\title{
SHAPE OPTIMIZATION FOR A NOISE REDUCTION PROBLEM BY NON-INTRUSIVE PARAMETRIC REDUCED MODELING
}

\author{
F. Bonizzoni ${ }^{1}$ and D. Pradovera ${ }^{2}$ \\ ${ }^{1}$ Augsburg University \\ Universitätsstraße 14, 86159 Augsburg, Germany \\ francesca.bonizzoniemath.uni-augsburg.de \\ ${ }^{2}$ CSQI - Institute of Mathematics - École Polytechnique Fédérale de Lausanne \\ Station 8, 1015 Lausanne, Switzerland \\ davide.pradoveradepfl.ch
}

Key words: Design optimization, model reduction, Helmholtz equation, acoustic scattering

\begin{abstract}
We study a PDE-constrained optimization problem, where the shape and liner material of the nacelle of an aircraft engine are optimized in order to minimize the noise radiated by the engine. More precisely, the acoustic problem is modeled by the Helmholtz equation with varying wavenumber $k$ on an exterior domain. A model reduction strategy is employed to alleviate the cost of the design optimization: the minimal rational interpolation technique is used to construct a surrogate (w.r.t. $k$ ) for the quantity of interest at fixed shape/material parameter values, and a parametric model order reduction approach is employed to combine surrogates at different shape/material designs, resulting in a nonintrusive methodology. Numerical experiments for shape and shape/material optimization are provided, to showcase the effectiveness of the presented methodology.
\end{abstract}

\section{INTRODUCTION}

In this paper we aim at minimizing the fan noise radiated from an aircraft engine exhaust, by optimizing (i) the shape of the nacelle of the aircraft engine and (ii) the impedance of the acoustic liner applied at the interior portion of the nacelle. This physical problem is modeled by a PDE-constrained optimization problem. In particular, we assume that the state problem fulfilled by the acoustic pressure $u$ is a linear scattering equation with wavenumber $k$ varying within a fixed interval of interest $K$, and we look for the minimum of the sound intensity at infinity, which we take as cost functional. Since the wavenumber $k$ varies, the optimal design depends on $k$.

The numerical solution of (parametric) PDE-constrained optimal control problems is often computationally very expensive, since it requires to solve the (parametric) state problem many times. To alleviate the computational cost, model order reduction (MOR) techniques [7, 11, 12, 13] may be used, where a surrogate for the quantity of interest (QoI) is constructed. For our purposes, the important features that the surrogate must possess are good approximation properties and fast evaluation. MOR methods are often split into two phases: an expensive offline phase is carried out, during which the sampling is performed and the surrogate of the QoI is assembled; the surrogate can then be cheaply evaluated at any (new) parameter value during the online phase. 
An analysis of the structure of parametric-in-frequency interior Helmholtz boundary value problems has been performed in [1], and then extended to scattering problems in [3]. There, the frequency response map, which associates to each wavenumber the corresponding solution to the Helmholtz boundary value problem, was proven to be meromorphic. In order to identify the pole-type critical values (i.e., the complex resonances of the system), we look for a surrogate in the class of rational functions (with respect to $k$ ). In the literature, we can find several strategies to build such approximations, e.g., Padé approximation [2,4], rational interpolation [10], and projection-based approaches [8]. In particular, in the present paper we employ the minimal rational interpolation technique introduced in [15] to get an approximation of the considered QoI w.r.t. $k$, at arbitrary (but fixed) designs.

In order to combine information at different shape/material values, a parametric model order reduction (pMOR) approach [14] is used, which allows to combine rational models over a (potentially highdimensional) parametric domain. We underline that the overall MOR approach is fully non-intrusive, allowing the use of a "black-box" solver for the computation of the input samples.

As will be shown numerically in Section 4, the QoI of the problem at hand is not convex and it also shows "localized" features with respect to the design parameters. This introduces additional complexity in the numerical search of its minimum over a given parameter domain, since numerical optimization algorithms might get stuck in local minima. Typically, this forces to explore the parametric domain in an almost brute-force fashion, to prevent any local features to go unnoticed. On top of this, we underline that the dependence of the state problem on shape and impedance parameters is not necessarily available explicitly, particularly in our non-intrusive framework. As such, it may be impossible to obtain

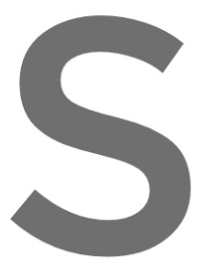
information on the derivatives of the Qol with respect
free optimization strategy.
Brute-force numerical optimization algorithms are
of the QoI requires the expensive solution of the s
surrogate for the QoI, rather than the QoI itself, al
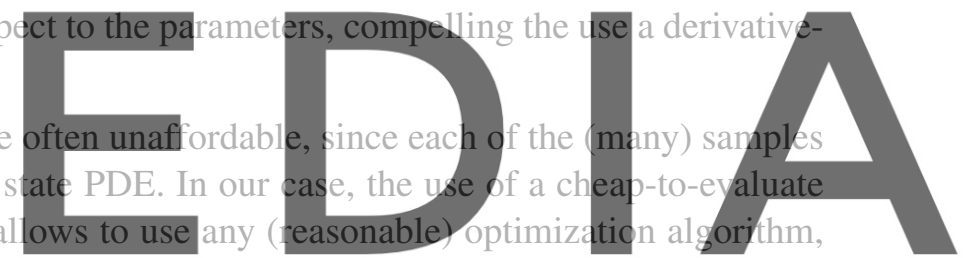

including the brute-force one. Note that, since the surrogate is known explicitly, derivative information

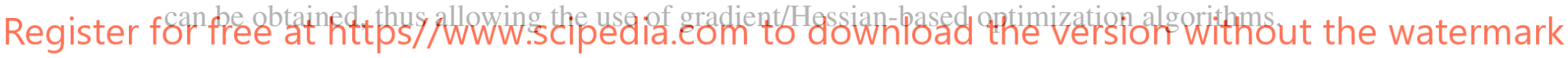

Approximate-then-optimize approaches have been considered before. For instance, we can find many examples of shape optimization problems in aeronautics solved by intrusive surrogate modeling methods, e.g., the reduced basis approach $[12,13]$. However, generally speaking, such methods are limited, since, on one hand, they cannot be applied in conjunction with black-box solvers, and, on the other hand, they rely on an affine dependence on the parameters, which is usually impossible to achieve (exactly) when geometric parameters are involved. This second issue can be overcome by constructing an affine approximation of the problem (e.g., by defining appropriate spatial warping maps [12], or by the empirical interpolation method [13]), with the resulting surrogate often having an increased complexity as a consequence, thus leading to a higher computational cost, both offline and, more importantly, online.

Non-intrusive approaches, e.g., those based on Gaussian process regression (GPR) [7, 11], are usually not affected by these two issues, and our proposed technique falls in this category. However, if one relies on a smooth kernel for the construction of the surrogate (as in GPR), difficulties may arise if the QoI displays sharp and localized features, as is the case here. In our approach, we employ rational (hence, locally non-smooth) functions to overcome this limitation. 
Outline of the paper. The rest of the paper is organized as follows. In Section 2 we introduce the parametric PDE-constrained optimal control problem. In Section 3 we describe the pMOR algorithm employed to construct a surrogate of the QoI. Numerical results are presented in Section 4 for two choices of design parameters. Finally, we draw our conclusions in Section 5.

\section{PROBLEM SETTING}

We consider a 3-dimensional region surrounding an engine exhaust, which we assume to be axisymmetric. We exploit this symmetry to pose the optimal control problem in a 2D domain, which was originally considered in [5] (where, however, the 3D domain displayed a planar symmetry instead of an axial one). The geometry of the domain $\Omega$ (in its reference configuration) is depicted in Figure 1, where $\Gamma_{N, 0}$ denotes the symmetry axis. The two boundary portions $\Gamma_{L}$ and $\Gamma_{N, 1}$ represent the boundary of the nacelle of the aircraft engine: the exterior boundary $\Gamma_{N, 1}$ is assumed to be perfectly reflective, whereas at the interior boundary $\Gamma_{L}$ an acoustic liner is applied to dampen engine noise. The noise source (which in the 3D problem is also axisymmetric) is located at the engine exhaust $\Gamma_{D}$. Moreover, the truncated computational domain $\Omega$ is assumed to be sufficiently large, i.e., $\Gamma_{\infty}$ is sufficiently far from the noise source, so that the Sommerfeld radiation condition at infinity can be approximated by a first order absorbing boundary condition on $\Gamma_{\infty}$. The nacelle angle $\theta \in \Theta \subset \mathbb{R}$ may vary, entailing a modification in the geometry of the domain. We refer to Figure 2 for a graphical depiction of the configurations corresponding to $\theta=0$ and $\theta=\frac{\pi}{8}$. In the following, to stress the dependence on $\theta$, we will denote the boundary of the nacelle and the domain by $\Gamma_{N, 1}(\theta), \Gamma_{L}(\theta)$, and $\Omega(\theta)$, respectively.
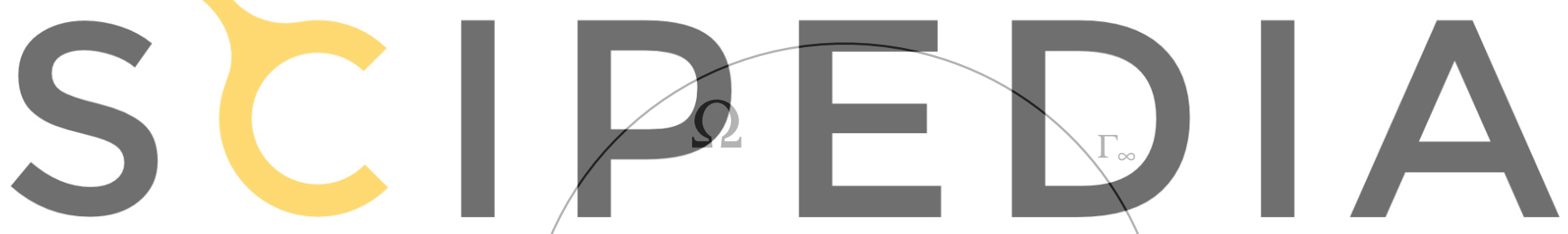

Register for free at https//www.scipedia.cons to ${ }^{N}$ lownload the version without the watermark

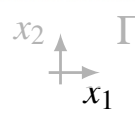

Figure 1: 2D geometry of the problem in the nominal configuration $\theta=0$. For reference, the length of $\Gamma_{D}$ is 1 . The $3 \mathrm{D}$ domain is obtained by a full revolution around $\Gamma_{N, 0}$.

We assume that the acoustic pressure $u$ fulfills the following non-dimensionalized Helmholtz boundary value problem

$$
\begin{cases}-\partial_{x_{1} x_{1}} u-\partial_{x_{2} x_{2}} u-\frac{1}{x_{2}} \partial_{x_{2}} u-k^{2} u=0 & \text { in } \Omega(\theta) \\ u=g & \text { on } \Gamma_{D} \\ \partial_{n} u=0 & \text { on } \Gamma_{N, 0} \cup \Gamma_{N, 1}(\theta) \\ \partial_{n} u+i v k u=0 & \text { on } \Gamma_{L}(\theta) \\ \partial_{n} u+i k u=0 & \text { on } \Gamma_{\infty}\end{cases}
$$

where $\left(x_{1}, x_{2}\right)$ denotes the spatial variable, cf. Figure 1 , and $n$ is the outer unit normal to the boundary (which, on $\Gamma_{N, 1}(\theta)$ and $\Gamma_{L}(\theta)$, depends on $\theta$ ). The somewhat unusual term $\frac{1}{x_{2}} \partial_{x_{2}} u$ arises when expressing 
the Laplace operator in cylindrical coordinates. Dirichlet boundary conditions are imposed on $\Gamma_{D}$, with $g$ representing the noise source, homogeneous Neumann boundary conditions are imposed on $\Gamma_{N, 0}$ and $\Gamma_{N, 1}(\theta)$, and Robin boundary conditions are imposed on $\Gamma_{L}(\theta)$. The complex number $v \in \Upsilon$ is the admittance of the liner, i.e., the reciprocal of the impedance factor $\chi$. As Dirichlet datum we take

$$
g\left(x_{2}\right)=\exp \left(\pi\left(\cos ^{2}\left(2 \pi x_{2}\right)-1\right)\right),
$$

cf. [5].

We are interested in optimizing the shape of the nacelle, as well as the liner impedance, so that the amount of noise that propagates to the far field is minimized. In particular, we will assume the wavenumber $k$ to vary in the interval of interest ${ }^{1} K \subset \mathbb{R}$, so that optimal shape and impedance will be dependent on $k$. As a measure of the acoustic noise (the QoI for our problem) we take $y: K \times \Theta \times \Upsilon \rightarrow \mathbb{R}^{+}$, defined as the (non-dimensional) total sound intensity at infinity (in the 3D domain). By a cylindrical change of variables and symmetry considerations, we can easily see that $y$ equals

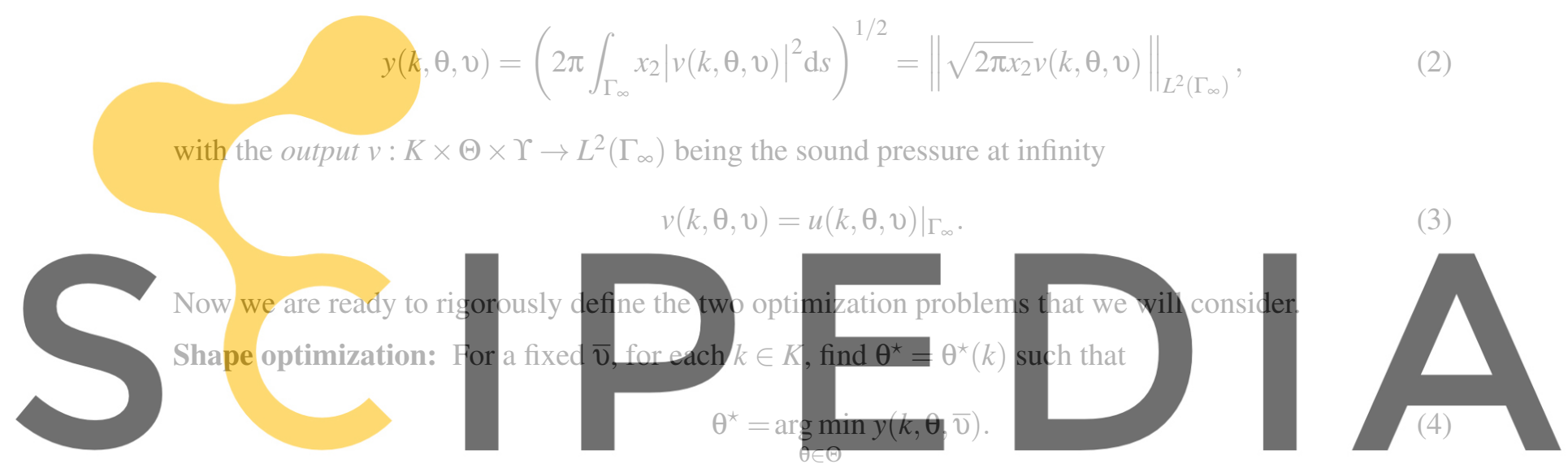

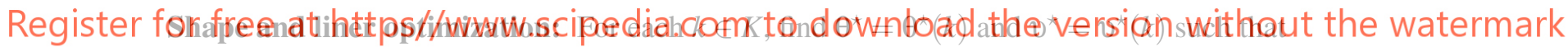

$$
\left(\theta^{\star}, v^{\star}\right)=\underset{\theta \in \Theta, v \in \Upsilon}{\arg \min } y(k, \theta, v) .
$$

\section{CONSTRUCTION OF THE SURROGATE QOI}

In this section, we will denote by $\xi \in \mathbb{R}^{P}$ and $\Xi \subset \mathbb{R}^{P}, P \geq 1$, a generic design parameter and parametric domain, respectively. This will allow us to describe a unified strategy to deal with both optimization problems (4) and (5) (for which, $\xi=\theta$ and $P=1$, and $\xi=(\theta, \operatorname{Re}(v), \operatorname{Im}(v))$ and $P=3$, respectively), as well as more general parameter-dependent optimization problems.

Due to the meromorphic structure of state problem (1), see [3], rational functions (with respect to $k$ ) represent the natural class where to look for an approximant of the solution $u$ for fixed values of $\xi$. In particular, we employ the minimal rational interpolation method introduced in [15]. To construct a global (i.e., dependent on both $k$ and $\xi$ ) surrogate for $u$ we rely on the pMOR approach introduced

\footnotetext{
${ }^{1}$ Our considerations extend naturally to $K \subset \mathbb{C}$, which may be of interest if wave attenuation is included in the model.
} 


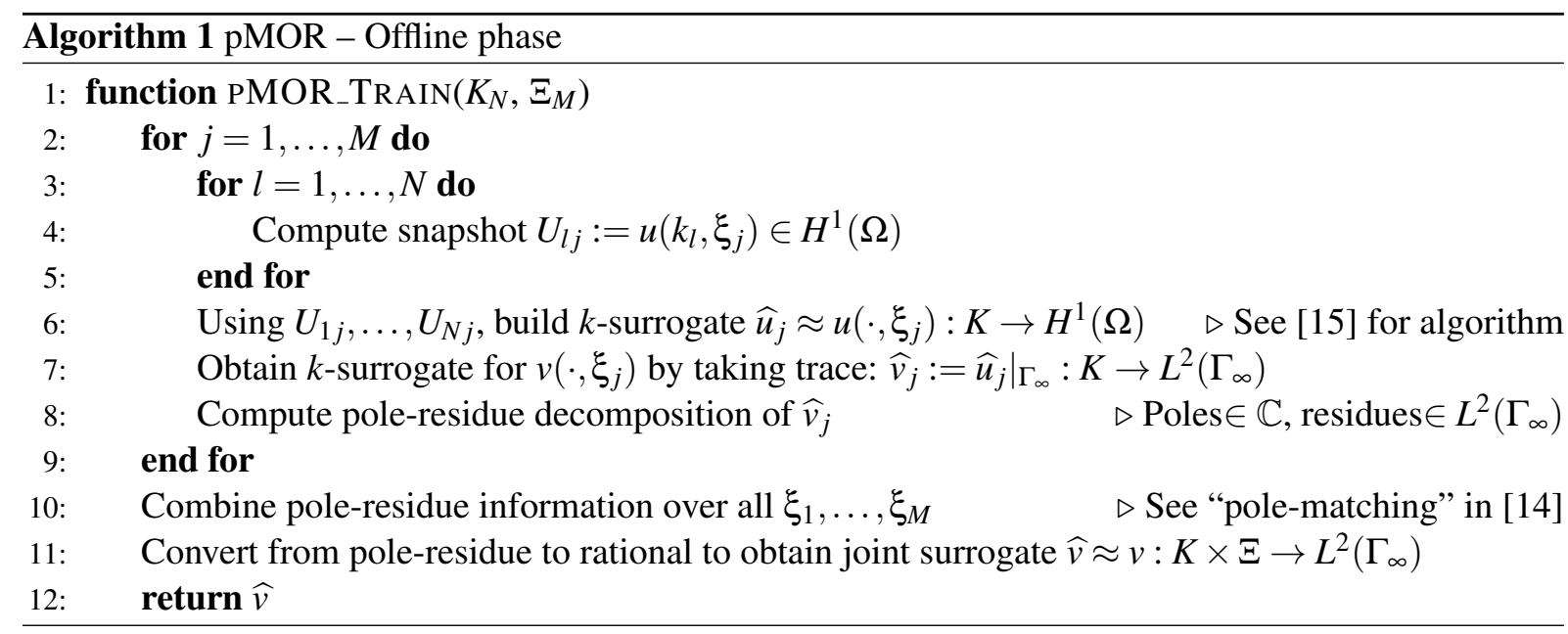

in [14], which provides a strategy for combining the rational approximants obtained for obtained for different training designs $\xi_{1}, \ldots, \xi_{M}$.

In the case at hand, the presence of geometric parameters entails variations in the spatial domain and, in turn, in its discretization. This causes issues in the construction of the joint surrogate of the solution $u$ itself, since approximants at different parameter values may be defined over different spatial meshes, thus making their (linear or nonlinear) combination not easily computable. However, since the output $v$

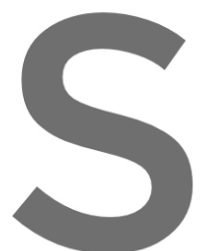
is the restriction of $u$ on combine information on $v$ Algorithm 1 summarizes gate for $v$. For simplicity, training parameter points both for $k$ and $\xi$, and we refer to [14] for a more detailed discussion on the topic.

Register fBerfice lat A the output $v$ is defined on the ( $\xi$-independent) exterior boundary $\Gamma_{\infty}$, rather than on the whole domain $\Omega(\theta)$. Hence, it is straightforward to combine residues $\widehat{v}_{j}$ for different values of $j$. We underline that, in practical applications, the space $L^{2}\left(\Gamma_{\infty}\right)$ will be replaced by a finite dimensional $(F E)$ subspace, which could potentially depend on $j$ because of different triangulations of $\Omega(\theta)$. If this is the case, it becomes important to restrict/extend all rational surrogates onto a common space, where they may be freely combined. For instance, one could consider the smallest FE subspace of $L^{2}\left(\Gamma_{\infty}\right)$ which contains all the residues: this corresponds to computing a new mesh for $\Gamma_{\infty}$, obtained by "overlaying" the different meshes for $\xi_{1}, \ldots, \xi_{M}$. In our numerical experiments this is not necessary, since, when constructing the numerical mesh, we force the FE degrees of freedom on $\Gamma_{\infty}$ to remain the same for all $\theta$.

The pMOR approach described above allows to build a surrogate for the output $v$ introduced in (3). From it, we can easily obtain a surrogate for the QoI $y$ defined in (2), by taking the (weighted) $L^{2}$ norm of $\widehat{v}$ on $\Gamma_{\infty}$ :

$$
\widehat{y}=\left\|\sqrt{2 \pi x_{2}} \widehat{v}\right\|_{L^{2}\left(\Gamma_{\infty}\right)}: K \times \Xi \rightarrow \mathbb{R}^{+} .
$$

The evaluation of the surrogate $\widehat{v}$ requires considerably less computational effort than computing the FE 


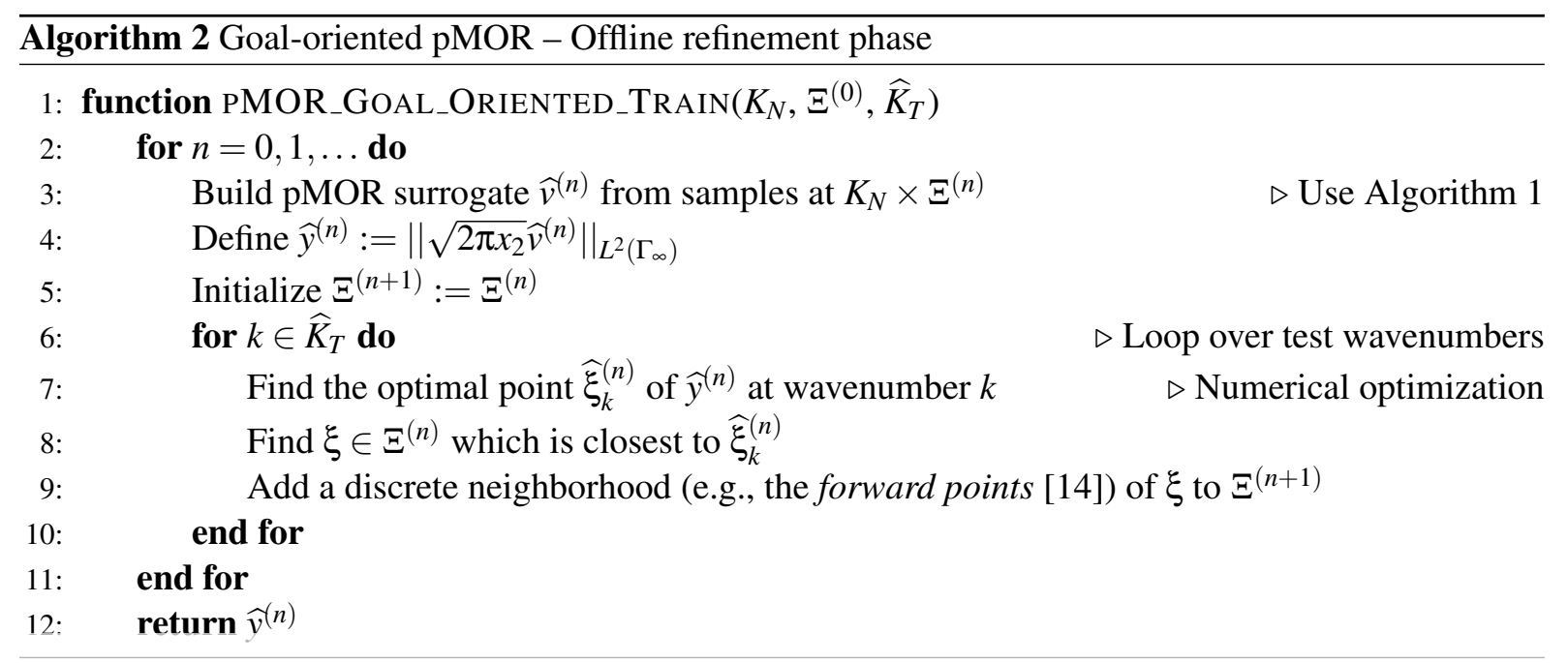

solution of (1), cf. the numerical examples in Section 4. In regard to the optimization problems (4)-(5), this has the beneficial consequence of allowing to use any reasonable numerical optimization algorithm for their solution. The choice of an efficient numerical optimizer is outside the scope of this paper. In our numerical tests in Section 4, the numerical optimization is performed by a brute-force approach, where the target function is sampled over a fine grid of parameters $\xi$, and their minimum is taken as solution. Of course, alternatives are possible.
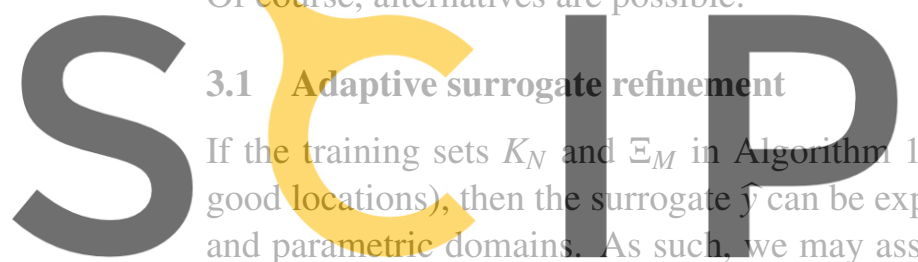
and parametric domains. QoI are properly identified. Moreover, the rational nature of the surrogate allows to pinpoint even sharp

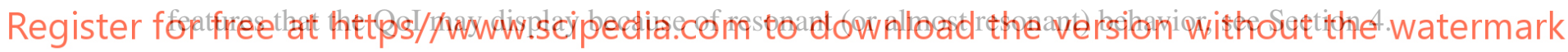

At the same time, in most applications, one might want to make the approximation of the the QoI sharper around the minima, for a finer tuning of the optimal design/control. To this aim, we detain here an offine goal-oriented surrogate refinement loop, which, given a reasonably accurate initial surrogate, tries to improve its approximation quality around the optimal points. Obviously, this comes at a non-negligible computational cost, since (potentially, many) new snapshots of the full order model have to be computed.

The procedure, which is summarized in Algorithm 2, relies on the definition of a set of $T$ test wavenumbers $\widehat{K}_{T} \subset K$, which need not have any relation to the training wavenumbers $K_{N}$. Every time a new surrogate is built, it is numerically optimized (e.g., by brute-force) at each of the test wavenumbers. Then sample points are added near all the $T$ newly computed optimal points. If the test set $\widehat{K}_{T}$ is "dense enough" 2 in $K$, this gives approximate information on the optimal design location over the whole $K$, allowing to better drive the sampling over $\Xi$.

To complete Algorithm 2, we must prescribe a termination criterion for the outer loop. In practice,

\footnotetext{
${ }^{2}$ Choosing a large $T$ is feasible, since the numerical optimization of the surrogate, which is repeated $T$ times at each refinement iteration, is quite cheap.
} 
the simplest option is setting a maximum number of refinement iterations. However, it is advisable to complement it with some goal-oriented "convergence tests", so that one may avoid oversampling. To obtain our numerical results, see Section 4, we have implemented the following two additional tests: given $\widehat{\xi}_{k}^{(n)}$, check if

(i) the optimal value has not changed much since the last iteration, i.e., in relative terms, if

$$
\left|\widehat{y}^{(n)}\left(k, \widehat{\xi}_{k}^{(n)}\right)-\widehat{y}^{(n-1)}\left(k, \widehat{\xi}_{k}^{(n-1)}\right)\right| \leq \varepsilon_{y} \widehat{y}^{(n-1)}\left(k, \widehat{\xi}_{k}^{(n-1)}\right)
$$

(ii) the optimal point has not changed much since the last iteration, i.e., if

$$
\left|\widehat{\xi}_{k}^{(n)}-\widehat{\xi}_{k}^{(n-1)}\right| \leq \varepsilon_{\xi}
$$

If either of these conditions is fulfilled, the local refinement of $\Xi^{(n+1)}$ near $\widehat{\xi}_{k}^{(n)}$ is skipped. Thus, we stop the outer loop if the maximum number of iterations is reached or if no refinement is performed.

\section{NUMERICAL RESULTS}

The numerical results in this section have been obtained through Python3 simulations run on the Helvetios cluster ${ }^{3}$ at EPFL. More specifically, the pMOR surrogate was constructed using the RROMPy package ${ }^{4}$. For each sampled value of $\theta$, the corresponding mesh is generated through Gmsh [9].

For a given $\theta$, the snapshots of $u$ are sought in the of $\Omega(\theta)$, with approximater

$\theta)$. The corresponding inet

4.1 Shape optimization

First, we consider as parameters the normalized

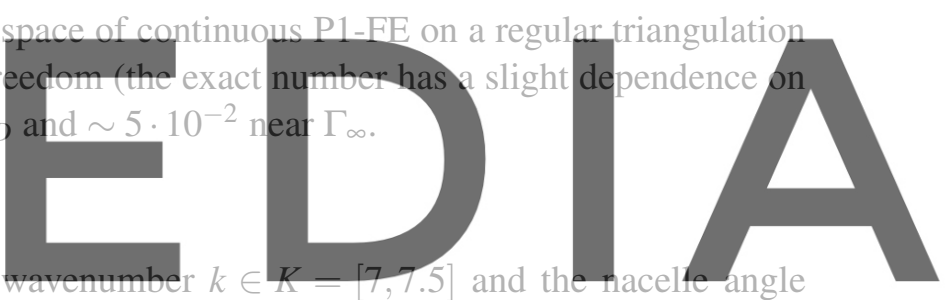

$\theta \in \Theta=\left[-\frac{\pi}{24}, \frac{\pi}{8}\right]$, while keeping the liner admittance fixed at $v=\bar{v}=0$ (perfect reflection on the whole

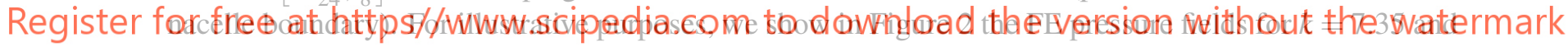
two different values of $\theta$.

We build a surrogate model $\widehat{y}: K \times \Theta \times\{\bar{v}\} \rightarrow \mathbb{R}^{+}$for the QoI by applying Algorithm 2, where

- the test set $\widehat{K}_{T}$ contains 11 equispaced points in $K$;

- the training wavenumber set $K_{N}$ is composed of the degree 10 Chebyshev nodes of $K(N=10)$;

- the initial training parameter set $\Xi^{(0)}$ contains 5 equispaced points in $\Theta$;

- the convergence tolerances $\varepsilon_{y}$ and $\varepsilon_{\xi}$ in (7)-(8) are set to $10^{-3}$ and $10^{-2}$, respectively.

The offline phase ends after 4 iterations of Algorithm 2. The final surrogate is based on 100 total FE snapshots, which explore 10 different geometries. See Figure 3 for more details on the $\Theta$-adaptivity. Therein, we can observe that in many cases no local refinement is performed despite the presence of one of the $\widehat{\xi}_{k}^{(n)}$, for some $k \in K_{T}$. This is because the point in question is "inactive" according to at least one of the termination criteria (7)-(8).

\footnotetext{
${ }^{3}$ epfl.ch/research/facilities/scitas/hardware/helvetios.

${ }^{4}$ Version 2.8.2. Source code available at c4science.ch/source/RROMPy.git.
} 

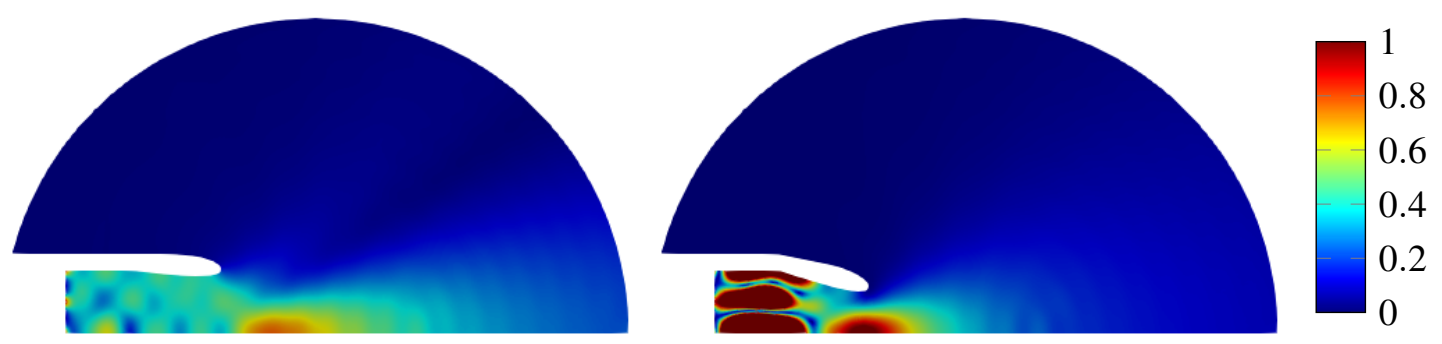

Figure 2: Plot of $|u|$ at $(k, \theta, v)=(7.35,0,0)$ (left) and $(k, \theta, v)=\left(7.35, \frac{\pi}{8}, 0\right)$ (right). In the right plot, the dark red regions near the engine are out of scale, reaching a maximum value of $\sim 7$. The corresponding values of the QoI are $y(7.35,0,0)=0.648$ and $y\left(7.35, \frac{\pi}{8}, 0\right)=0.494$.

We show in Figure 4 the restriction of $\widehat{y}^{(n)}$ to $k=7.25$, for $n=0$ (initial coarse surrogate) and $n=3$ (final surrogate). In particular, we highlight the presence of a local effect due to resonance (the singular behavior close to 0 ) and the non-convexity of the QoI (demonstrated by the presence of local maxima).

The surrogate can be evaluated at a new frequency/design point $(k, \theta)$ in $\sim 0.25 \mathrm{~ms}$ (average over $\sim 10^{4}$ samples), whereas solving once the full order FE model takes $\sim 1.3 \mathrm{~s}$ (average over $\sim 10^{4}$ samples, not including meshing). This allows for a significant speed-up in the online optimization phase. The results of the optimization using the computed surrogate are shown in Figure 5, where the optimal design is computed at 101 equispaced wavenumbers in $K$. As desired, the QoI is uniformly lower in the optimized configuration. The improvement is only minor for small and large values of $k$ (i.e., the QoI in the nominal configuration is close to the optinal value for thes
noticeable. We also include some validation point
the nominal configuration $\theta=0$, as well as in the opt
optimization of the surrogate. The agreement betw
As a qualitative comparison, in Figure 6 we comparte
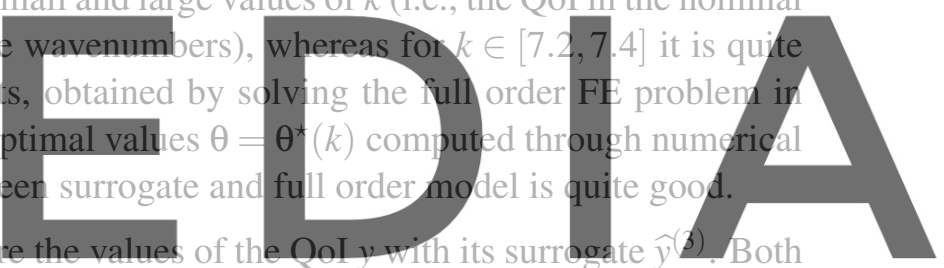

functions were evaluated on a tensor grid in the set $K \times \Theta$. However, since the surrogate is considerably

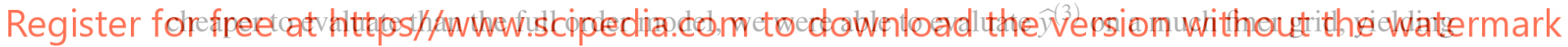
a significantly more detailed plot. In particular, the surrogate allows to detect a so-called resonance line (the sharp feature in the right plot) which could not have been identified by relying only on coarse sampling of the full order model. This also gives a justification for the use of rational-based surrogates for problems with (quasi-)resonant behavior, in contrast to commonly used non-intrusive techniques for "smooth" problems, e.g., kernel methods like kriging [11] and RBFs [6].

A direct comparison between Figure 5 and Figure 6 shows that, for $k \in[7.2,7.4]$, the noise reduction is achieved by setting the optimal nacelle angle $\theta^{\star}$ in such a way that the resulting configuration is almost at resonance. This means that the resonant mode is used to "trap" acoustic energy near the exhaust, instead of letting it escape towards $\Gamma_{\infty}$.

\subsection{Shape and liner optimization}

Given the satisfactory results from the previous section, here we enlarge the wavenumber range and we add 2 more parameters to the problem, namely the real and imaginary parts of the liner admittance $v=v_{R}+i v_{I}$ (sometimes referred to as conductance and susceptance, respectively). In particular, we take $k \in K=[7,8], \theta \in \Theta=\left[-\frac{\pi}{24}, \frac{\pi}{8}\right], v_{R} \in \Upsilon_{R}=[0,1]$, and $v_{I} \in \Upsilon_{I}=[0,1]$. We define the (complex) 


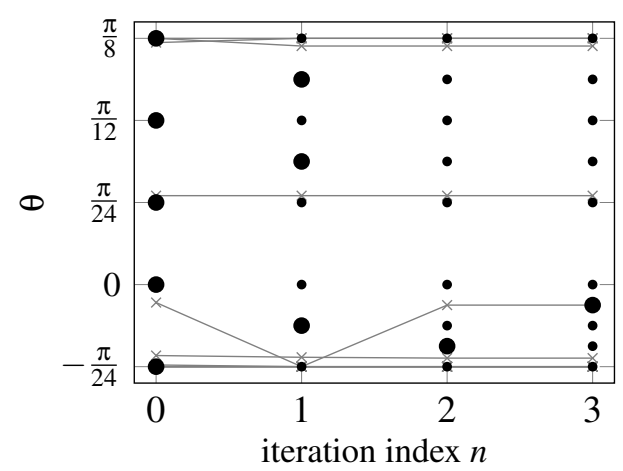

Figure 3: Offline sampling history. Large dots are points added at the $n$-th iteration; small dots are carried over from the previous iterations. The gray lines are $\left\{\hat{\xi}_{k}^{(n)}\right\}_{k \in K_{T}}$.

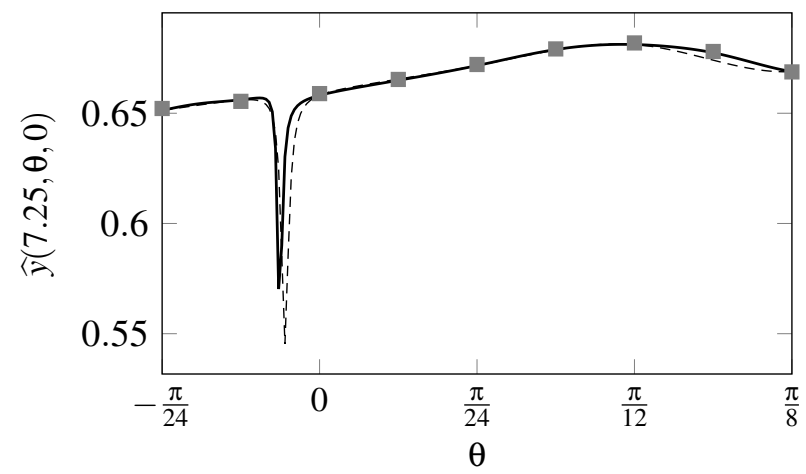

Figure 4: Coarse initial surrogate $\widehat{y}^{(0)}$ (dashed line) and refined final surrogate $\widehat{y}^{(3)}$ (full line) for $k=7.25$. The gray squares are samples from the full order model $y$.
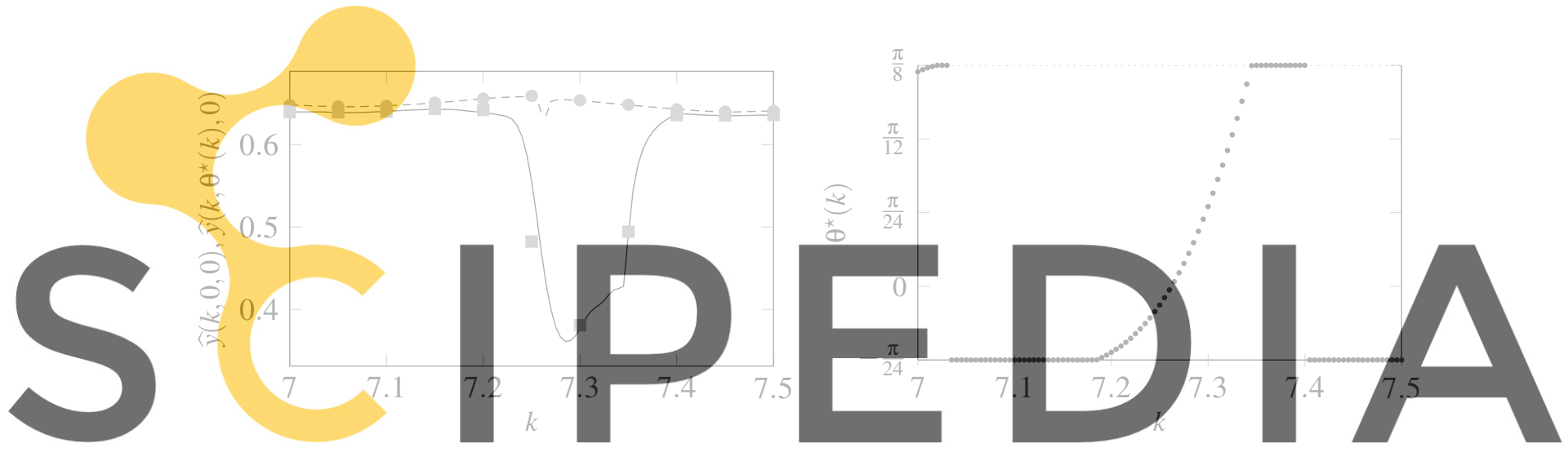

Figure 5: On the left, surrogate QoI in the nominal $\theta=0$ (dashed line) and optimized $\theta=\theta^{\star}(k)$ (full

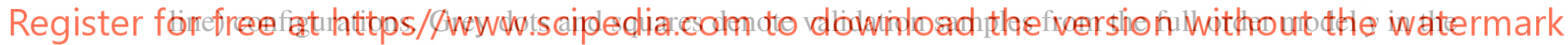
nominal and optimized cases, respectively. On the right, the surrogate optimal point $\theta^{\star}(k)$.
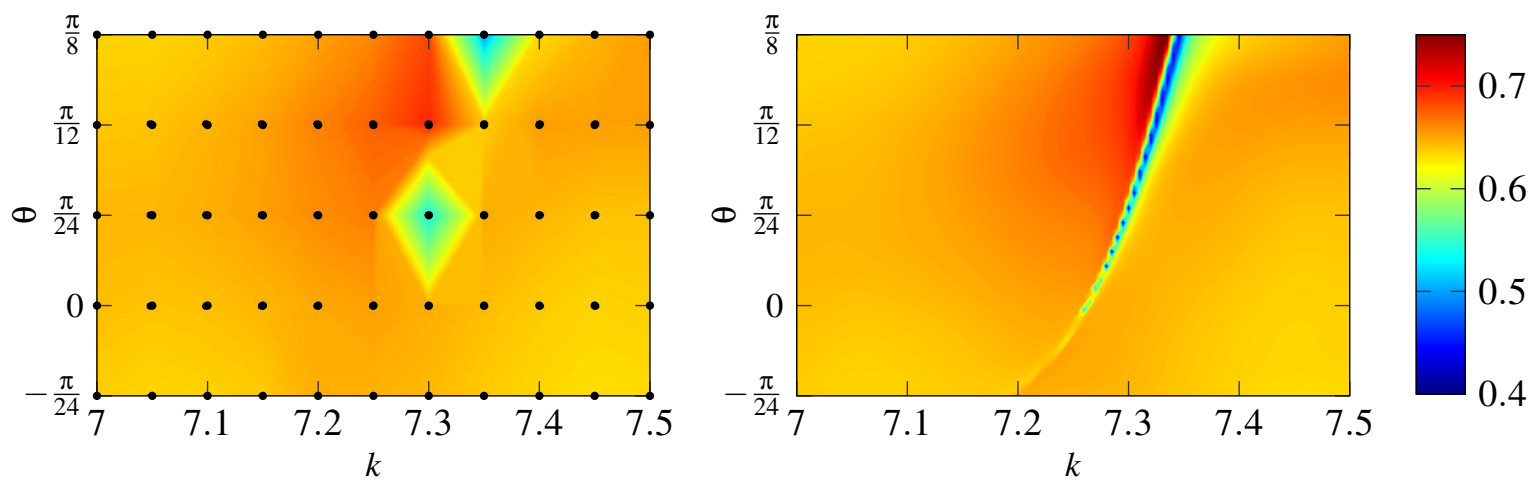

Figure 6: Plot of $y$ (left) and $\widehat{y}^{(3)}$ (right). The color scale is the same for both plots. In the left plot, the color is interpolated from samples at the 55 points in the grid of black dots. 
admittance domain as $\Upsilon=\left\{v_{R}+i v_{I}\right\}_{v_{R} \in \Upsilon_{R}, v_{I} \in \Upsilon_{I}}$.

We build a surrogate model $\widehat{y}: K \times \Theta \times \Upsilon \rightarrow \mathbb{R}^{+}$for the QoI by applying Algorithm 2, where

- the test set $\widehat{K}_{T}$ contains 11 equispaced points in $K$;

- the training wavenumber set $K_{N}$ is composed of the degree 20 Chebyshev nodes of $K(N=20)$;

- the initial training parameter set $\Xi^{(0)}$ is the sparse grid of depth 2 of $\Theta \times \Upsilon_{R} \times i \Upsilon_{I}$, which contains 25 points, see [14] and Figure 7;

- the convergence tolerances $\varepsilon_{y}$ and $\varepsilon_{\xi}$ in (7)-(8) are both set to $10^{-2}$.

The offline phase ends after 10 iterations of Algorithm 2. The final surrogate is based on 1860 total FE snapshots, which explore 93 points in the set $\Theta \times \Upsilon$, involving 10 different geometries and 42 different admittance values. Due to the increased dimension of the parameter space, a fairly high amount of snapshots of the full order model is required to build the surrogate.

We report the locations of the sample points in Figure 7, where we can observe a high concentration of samples for small values of $v_{R}$ and $v_{I}$. This is in agreement with what we obtain in the online phase, where the minimizer for the surrogate QoI is computed at 101 equispaced wavenumbers in $K$. Indeed, the optimal values $v_{R}^{\star}(k)$ and $v_{I}^{\star}(k)$ are below average for all $k \in K$, see Figure 9 (left) and (center).

As in the previous numerical test, we observe that the QoI is uniformly lower in the optimized configuration, see Figure 8. However, the improvement is much more pronounced than in the previous example. By looking at the optimal design in Figure 9, we can observe that an admittance $v$ of approximately

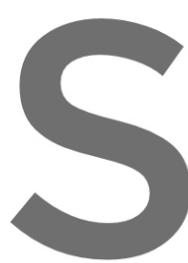
$0.25+0.4 i$ seems to work well over the whole wa
higher variability. We depict in Figure 10 the FE
wavenumber $k=7.35$.
Also in this case, we achieve a significant speed-
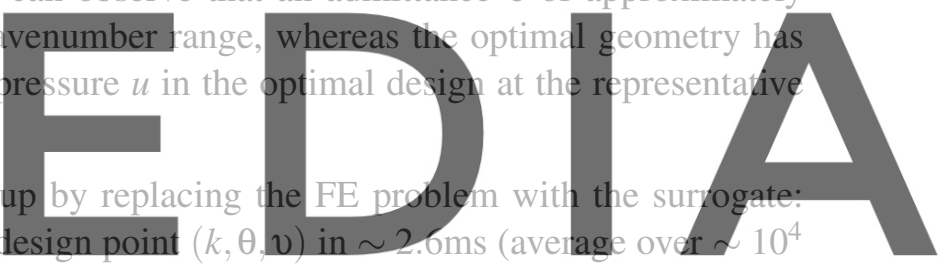

samples), whereas solving once the full order FE model takes $\sim 1.3 \mathrm{~s}$ (average over $\sim 10^{4}$ samples, not

Register forffidę atephetgs//www.scipedia.com to download the version without the watermark

\section{CONCLUSIONS AND OUTLOOK}

We have presented a non-intrusive model order reduction method based on rational approximation, which can be used to construct a surrogate for the intensity of the noise radiated by an aircraft engine. The surrogate is then minimized to identify the optimal value of the design parameters (geometry and material) for all values of the wavenumber $k$ varying within an interval of interest. Our numerical experiments have confirmed the effectiveness of the proposed methodology.

Considering the positive results in the considered 2D example with 2 design parameters in addition to the wavenumber, we believe our approach to be appropriate also for 3D scattering problems with a higher number of design parameters, e.g., with a more refined parametrization of the geometry, so that not only the nacelle angle, but also other features of the nacelle, may be optimized. In particular, we remark that a MOR-based design optimization strategy like the one we propose may be even more beneficial for 3D problems, thanks to the potential for an even more dramatic speed-up. 


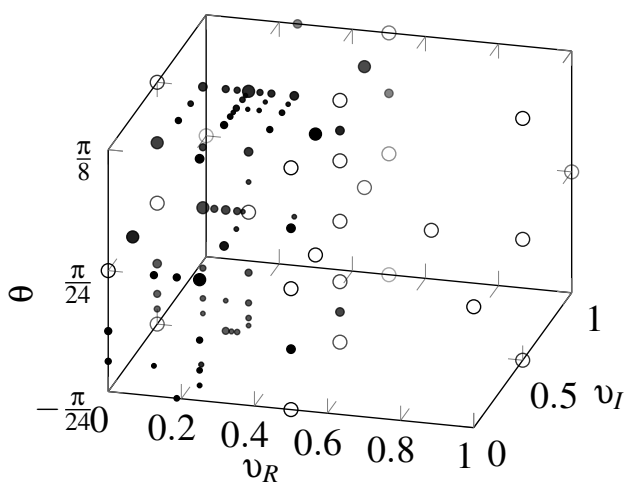

Figure 7: Sampling points in the offline training. Points added at later iterations have progressively smaller size. The initial sampling set is denoted by hollow circles.

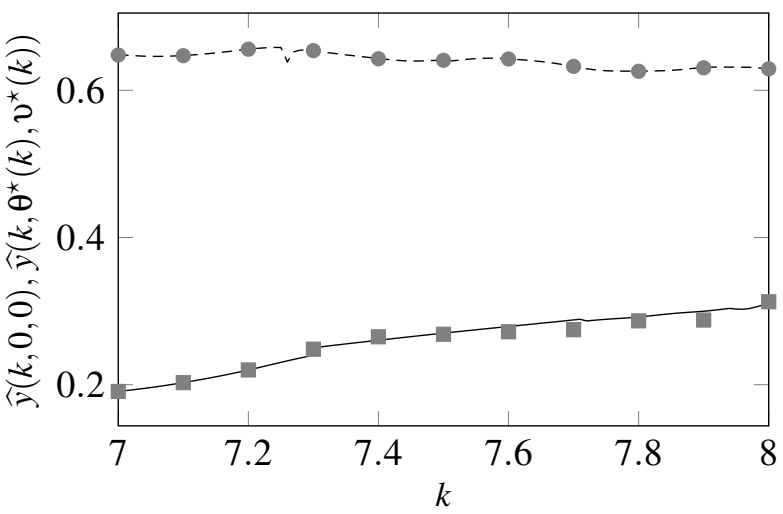

Figure 8: Surrogate QoI in the nominal $(\theta, v)=(0,0)$ (dashed line) and optimized $(\theta, v)=\left(\theta^{\star}(k), v^{\star}(k)\right)$ (full line) configurations. Grey dots and squares denote validation samples from the full order model $y$ in the nominal and optimized cases, respectively.
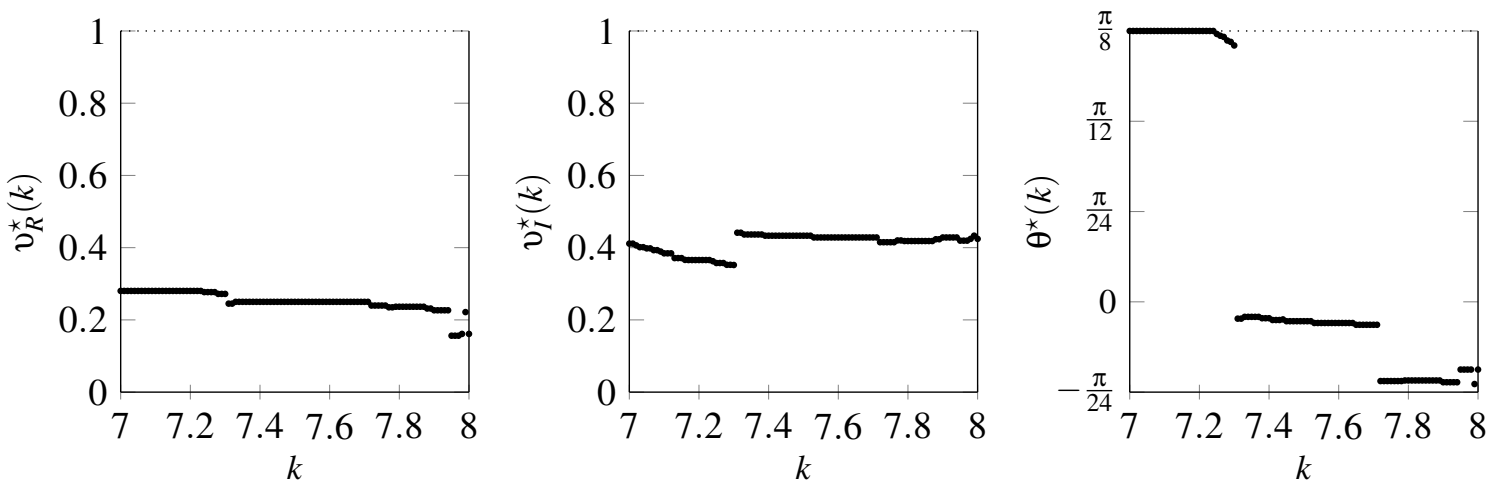

Figure 9: Optimal values of $v_{R}$ (left), $v_{I}$ (center), and $\theta$ (right) found by optimizing the surrogate.

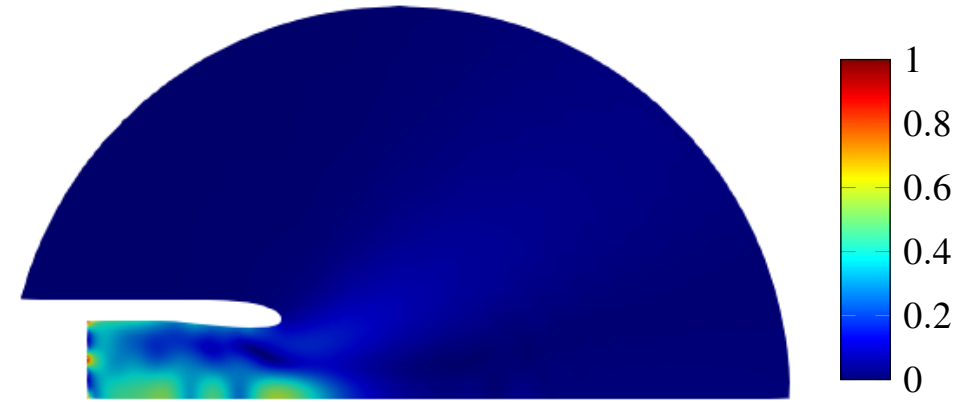

Figure 10: Plot of $|u|$ at $(k, \theta, v)=(7.35,-0.0218,0.25+0.436 i)$. The corresponding value of the QoI is $y(7.35,-0.0218,0.25+0.436 i)=0.261$. See Figure 2 (left) for the solution in the nominal configuration. 


\section{ACKNOWLEDGMENTS}

FB acknowledges partial financial support from the Austrian Science Fund (FWF) through project F 65, and from the FWF Firnberg-Program, grant T998. DP acknowledges financial support from the Swiss National Science Foundation (SNF) through project 182236.

\section{REFERENCES}

[1] Bonizzoni, F., Nobile, F., and Perugia, I. Convergence analysis of Padé approximations for Helmholtz frequency response problems. ESAIM: M2AN (2018) 52:1261-1284.

[2] Bonizzoni, F. and Pradovera, D. Distributed sampling for rational approximation of the acoustic scattering of an airfoil. Proc. Appl. Math. Mech. (2019) 19.

[3] Bonizzoni, F., Nobile, F., Perugia, I., and Pradovera, D. Least-Squares Padé approximation of parametric and stochastic Helmholtz maps. Adv. Comput. Math. (2020) 46.

[4] Bonizzoni, F., Nobile, F., Perugia, I., and Pradovera, D. Fast Least-Squares Padé approximation of problems with normal operators and meromorphic structure. Math. Comp. (2020) 89:1229-1257.

[5] Cao, Y., Hussaini, M.Y., and Yang, H. Estimation of optimal acoustic liner impedance factor for reduction of radiated engine noise. Int. J. Numer. Anal. Model. (2007) 4:116-126.

[6] Chen, W., Hesthaven, J.S., Bai, J., Qiu, Y., Yang, Z., and Tihao, Y. Greedy nonintrusive reduced order model for fluid dynamics. AIAA J. (2018) 56:4927-4943.

[7] Ding, F. and Kareem, A. A multi-fidelity shape optimization via surrogate modeling for civil structures. J. Wind Eng. Ind. Aerodyn. (2018) 178:49-56.

[8] Benner, P. and Feng, L. A Robust Algorithm for Parametric Model Order Reduction Based on Implicit Moment Matching. In Reduced Order Methods for Modeling and Computational Reduction (2014) 159-185.

[9] Geuzaine C. and Remacle J.-F. Gmsh: a three-dimensional finite element mesh generator with built-in pre- and post-processing facilities. Int. J. Numer. Methods Eng. (2009) 79.

[10] Ionita, A.C. and Antoulas, A.C. Data-driven parametrized model reduction in the Loewner framework. SIAM J. Sci. Comput. (2014) 36:A984-A1007.

[11] Keane, A.J. and Voutchkov, I.I. Robust design optimization using surrogate models. J. Comput. Des. Eng. (2020) 7:44-55.

[12] Lassila, T. and Rozza, G. Parametric free-form shape design with PDE models and reduced basis method. Comput. Methods Appl. Mech. Eng. (2010) 199:1583-1592.

[13] Manzoni, A., Quarteroni, A., and Rozza, G. Shape optimization for viscous flows by reduced basis methods and free-form deformation. Int. J. Numer. Methods Fluids (2012) 70:646-670.

[14] Nobile, F. and Pradovera, D. Non-intrusive double-greedy parametric model reduction by interpolation of frequency-domain rational surrogates. arXiv:2008.10864.

[15] Pradovera, D. Interpolatory rational model order reduction of parametric problems lacking uniform inf-sup stability. SIAM J. Numer. Anal. (2020) 58:2265-2293. 